\title{
Gait disturbances in the acute medically ill elderly
}

\author{
M.J. Steiger and P. Berman \\ Department of Health Care of the Elderly, City Hospital, Nottingham, UK
}

Summary: The characteristics of acute deterioration in truncal movements, posture and gait were studied in patients admitted with acute non-neurological disease. Reversible gait abnormalities with characteristics of a 'lower-half parkinsonism' were associated with a disorder of axial movement, disability (as assessed by an activities of daily living score), and low mental test score. There was no association of gait dysfunction with tests of upper limb apraxia.

An 'at risk' sub-group of elderly patients was identified who during an acute illness lose their walking independence, of whom some also have great difficulty performing axial movements. Furthermore, any assessment of locomotor and axial movement in elderly patients must consider that any impairment may be the result of an acute medical illness.

\section{Introduction}

Disorders of gait are a major cause of morbidity among elderly persons, contributing to the risk of disabling injury. ${ }^{1}$ The aim of this study was to identify whether disintegration of gait can be an acute phenomenon in the ill elderly. We documented the characteristics of the gait, and the relationship to other motor tasks in acutely ill elderly patients admitted with non-musculoskeletal or neurological disease. We observed whether the features of the gait changed in the time from admission until discharge of the patient, and if the changes were accompanied by alterations of performance in other motor tasks and in activities of daily living.

\section{Methods}

\section{Subjects}

A total of 189 consecutive patients admitted acutely to a general ward under the care of one physician were considered for entry into the study. Of these 113 patients were excluded (Table I). Patients with mental impairment in the absence of other neurological signs were included. The 76 patients accepted into the study (mean age 80.6, s.d. 7.0 years, 17 males) were assessed within 48

Correspondence: M.J. Steiger, M.R.C.P., Department of Clinical Neurology, National Hospital for Neurology and Neurosurgery, Queen Square, London WCIN 3BG, UK.

Accepted: 27 May 1992 hours of admission, at one week, and at discharge. The patients were compared to 90 elderly controls who were consecutive visitors to the hospital (mean age 75.1 , s.d. 5.0 years, 38 males). Control subjects fulfilled the same entry criteria as the patients in not having neurological or rheumatological disease.

In the patient group, the present diagnosis was obtained from history and examination, and significant past medical history and home circumstances were verified from medical records, relatives and/or general practitioners.

\section{Assessments}

The assessment included:

1. A simple ten item test of mental state. ${ }^{2}$ Scores below seven suggest mental impairment due to confusional state or dementia.

Table I Exclusion criteria in 113 of 189 consecutively admitted patients

Criteria $\begin{gathered}\text { Number } \\ \text { of patients }\end{gathered}$

Neurological disease 56

Orthopaedic/rheumatological

25

Acute myocardial ischaemia/infarction $\quad 6$

Blind (unable to count fingers)

Severe hearing deficit

Death prior to assessment

In-hospital transfer

Refused

Self-discharge 
2. All subjects were rated for degree of disability using an activities of daily living scale (Barthel) ${ }^{3,4}$ The range of possible scores are from $0 / 20$ representing severe, dependent disability to 20/20 representing full independence.

3. We examined each subject for different forms of apraxia, to identify a relationship between apraxic errors and abnormalities of axial movements. Using six simple upper limb movements, subjects were assessed for evidence of ideomotor apraxia, defined as a failure to perform a requested movement adequately. ${ }^{5}$ Ideational apraxia, defined as impairment of limbs to carry out a sequence of movements ${ }^{5}$ was similarly assessed using paradigms suggested by Brown ${ }^{6}$ (Table II). Patients had to be able to perform component movements. We also looked for evidence of a constructional apraxia with a simple constructional task (Table II).

4. Tests for disordered axial movement were similar to those of Cox et al. ${ }^{7}$ They included turning over in bed through $90^{\circ}$ in each direction, rising up from supine to the sitting position, and rising from a chair to standing. Each component was scored, but only added to the axial apraxia score if the goal of the movement was achieved (Table III).

5. In subjects able to stand, postural stability was assessed by the simple Romberg's test. ${ }^{8}$ We timed how long each subject could balance on one foot, and recorded the best of three attempts. When examining gait characteristics, subjects were allowed to use a walking aid to optimize balance and walking efficiency. Any aid to assist in walking was recorded. Subjects were assessed for hesitation in initiating gait, posture during walking, heel strike and toeing off during the walking cycle, and presence of normal tandem gait.

Table II Tests of upper limb apraxia

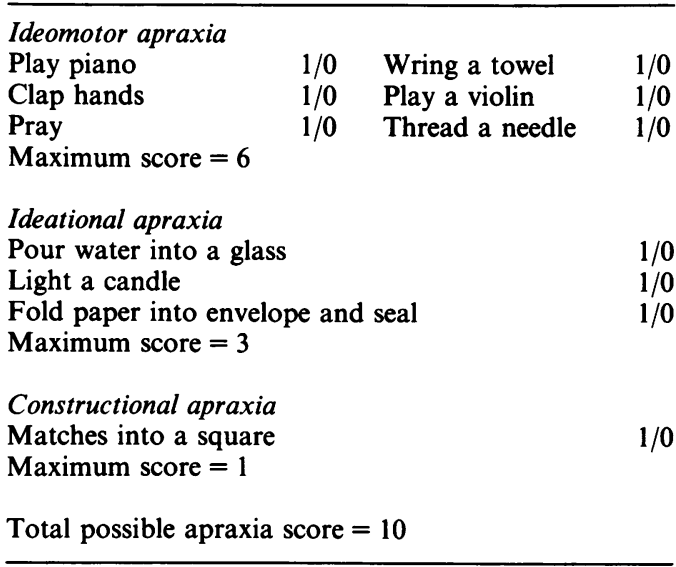

Table III Axial movement score

Turn over in bed

Right - swing arm $\quad 1 / 0 \quad$ Flex leg $\quad 1 / 0$

$\begin{array}{llll}\text { Left }- \text { swing arm } & 1 / 0 & \text { Flex leg } & 1 / 0\end{array}$

Maximum score $=4$

Sit up from horizontal

Place and flex arms

Flex trunk

Flex legs

Maximum score $=3$

Get out of chair (chair with arms)

Feet under chair

Lean forward

Push with hands

Maximum score $=3$

Total possible axial movement score $=10$

\section{Statistical methods}

Within the patient group, change across the assessment occasions was examined using repeated measures analysis of variance. Differences between the patients and controls or between subgroups of patients were examined with independent $t$-tests The association between variables was assesse with Pearson correlation coefficients. Categorica $\bar{b}$ data were analysed with a chi-square test. For alP analyses, $P$ values less than or equal to 0.01 were considered significant.

\section{Results}

Of the 76 patients who entered the study and were assessed on admission, six subsequently died: two from pneumonia, and one each from chronic myeloid leukaemia, disseminated breast carcinoma, liver metastases at an unknown primary site and pulmonary embolus.

\section{Patients vs controls}

All the control subjects were able to walk normally without the use of a walking aid, except one individual who required the use of a stick. This contrasts to the walking ability of the patients described in Table IV. The high mean Barthel (19.7, s.d. 0.8) of the control group suggests that elderly visitors to hospital are fully independent.

The control elderly population of successive visitors to the hospital were not age- or sexmatched to the patient group. However, the distribution of males and females was not significantly different in the two patient groups distinguished on the basis of axial movement scores above five, or 
Table IV Results of patients

\begin{tabular}{lccc}
\hline & $\begin{array}{c}\text { Admission } \\
(\mathrm{n}=76)\end{array}$ & $\begin{array}{c}\text { One week } \\
(\mathrm{n}=70)\end{array}$ & $\begin{array}{c}\text { Discharge } \\
(\mathrm{n}=70)\end{array}$ \\
\hline Axial movement score $(<6 / 10)$ & $10(2)$ & 2 & 2 \\
Unable to walk despite assistance & $5(2)$ & 1 & - \\
Apraxia score $(<10 / 10)$ & $14(3)$ & 12 & 11 \\
In subjects able to walk $(\mathrm{n}=71):$ & & & \\
Walk with assistance & $11(1)$ & 6 & 1 \\
Walk with aid (for example, stick) & 13 & 13 & 22 \\
Walk without aid & $47(1)$ & 27 & 47 \\
Unable to complete 10 m & $5(1)$ & 4 & - \\
Start hesitation & $9(1)$ & 9 & 3 \\
Loss of tandem gait & 13 & 11 & 2 \\
Loss of heel strike & $17(1)$ & 15 & 10 \\
Unable to stand on one foot & $22(4)$ & 8 & 6 \\
Rombergism & $13(1)$ & 7 & 1 \\
\hline
\end{tabular}

Figures in brackets represent patients who subsequently died and are included in admission score only. Patients discharged at/or less than one week do not have a one week assessment, but have a discharge assessment.

equal to or below five (disordered axial movement, DAM). Similarly there was no association between the sex of the patient and independence in walking on admission. Furthermore, there were no differences in age between patients who had or did not have independence in walking at admission (mean age of patients with loss of walking independence $=82.2$, s.d. 8.6 years, mean age of patients able to walk independently $=80.2$, s.d. 6.5 years). The age of the two groups with axial movement scores above five, or equal to or below five were also not different (mean age of patients in disorder of axial movement group (DAM) $=83.8$, s.d. 8.1, with mean age $=80.1$, s.d. 6.7 in the non-DAM group).

Only two of the 90 subjects in the control group scored less than $7 / 10$ on the test of mental state, compared with 27 patients on admission. The mental test score on admission was significantly associated with that at discharge suggesting that patients were retaining their admission allocation at discharge $(r=0.84 ; P<0.001)$. Twenty-seven of the 76 patients $(35.5 \%)$ scored less than $7 / 10$ on the mental test score on admission, compared with 20 of the 70 patients $(28.5 \%)$ at discharge. Of the two control subjects with a low mental test score, one was unable to turn over in bed or sit up from lying supine, but had a normal gait. The other walked normally and was able to perform all axial tasks normally.

Only one other control subject was unable to perform an axial movement. This was a 68 year old man who walked normally, but was unable to sit up from lying supine. He scored seven out of 10 on the mental test score. The other control subjects were able to perform each axial manoeuvre, but 10 subjects adopted an unusual method in achieving the final position, for example, when attempting to sit up, one subject lifted both legs in the air then threw them forwards whilst elevating the trunk. Other more common examples included failing to swing the arm and flexing the legs when turning over, and/or not using the arms to help in sitting up from supine.

\section{Axial/truncal disorders}

Ten patients (13.2\%) had a severe disorder of axial movement with an inability to perform one or more tasks, resulting in a score of five or less out of 10 . Their axial disorders are described in Table $\mathrm{V}$. Presence of DAM was associated with loss of walking independence $(P<0.001)$. On admission, only two of the 10 patients with DAM were able to walk independently, and three were unable to walk despite assistance. The gait of these patients with DAM was characterized by start hesitation and small steps with an increase in double support time. In only one of the 10 DAM patients was there a history of falls, implying that the poverty of gait was not a result of previous falls and resulting poor confidence.

Two of the patients with DAM died, but a striking feature in six of the surviving eight was the recovery of normal axial movement, and partial recovery in the other two. This improvement was statistically significant (mean axial score on admission in DAM group $=4.6$, s.d. 0.5 , and on discharge $=8.5$, s.d. $1.6, P<0.001$ ). The gait improved in all DAM patients with a return to normal in four, and independent walking in all the eight 


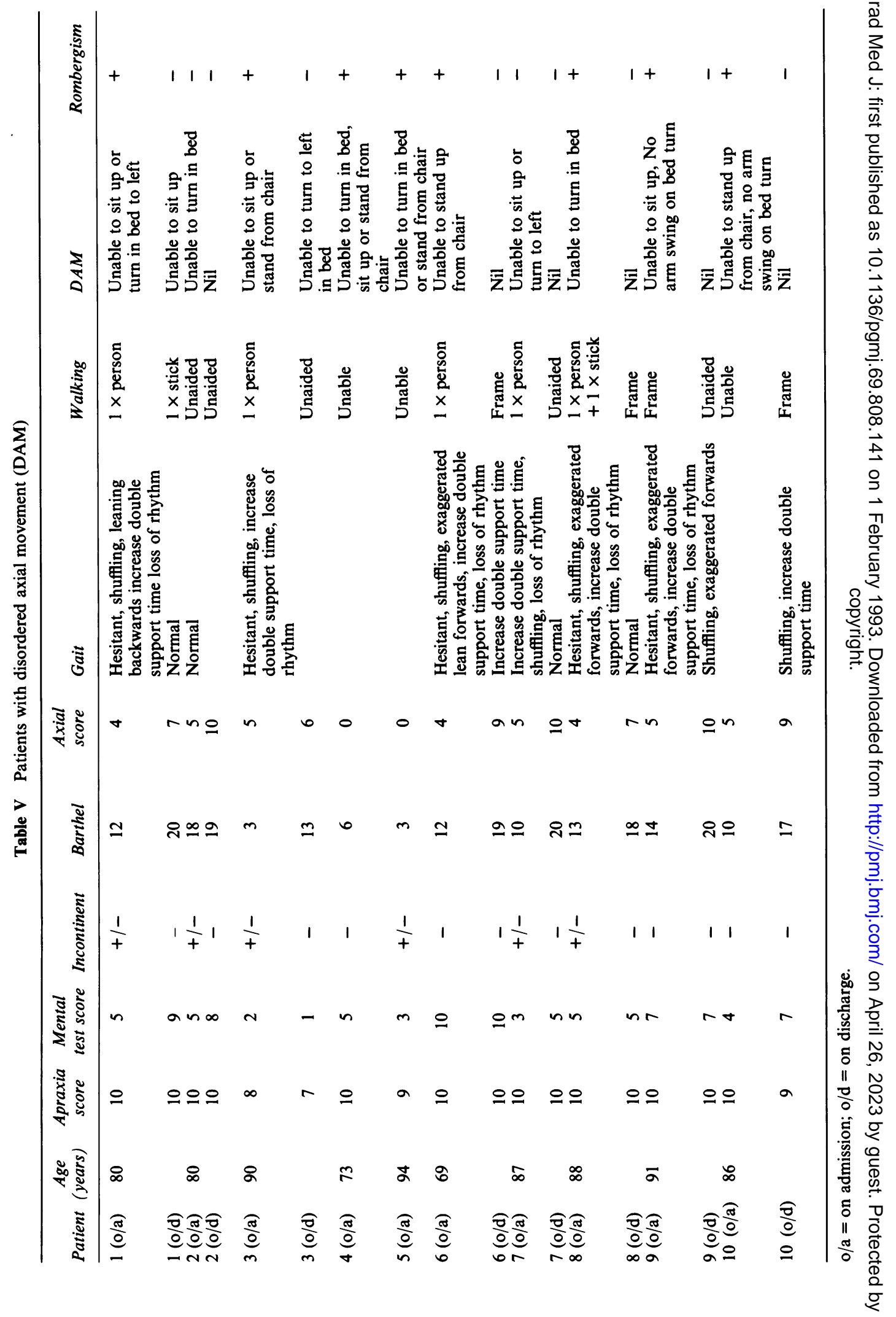


survivors. The improvement of gait and the loss of Rombergism at discharge argues against the existence of a previous severe gait disturbance prior to admission.

The patients with DAM on admission were also significantly more disabled (lower Barthel scores) compared to the non-DAM group (Barthel on admission in DAM group $=10.3$, s.d. 4.5, Barthel in non-DAM group $=17.4$, s.d. $3.2, P<0.01$ ). However, at discharge there were no significant differences in the disability as indicated by the Barthel scores between the patients with or without DAM on admission (Barthel of DAM group at discharge $=18.3$, s.d. 2.9 , Barthel of non-DAM group $=19.3$, s.d. 1.7).

The ability of patients with DAM to regain independence is indicated by their placement at final discharge. The proportion of patients in the DAM group admitted to residential care (one of eight patients) was no greater than in the nonDAM group (four of 62 patients), suggesting that these patients were able to continue to live in the community.

A greater proportion of the patients in the DAM than in the non-DAM group scored less than the cut-off $(7 / 10)$ on the mental test score (mean score in DAM group $=4.9$, s.d. 2.3 , and non-DAM group $=7.2$, s.d. $2.4, P<0.01$ ).

Only one patient failed to obtain full marks on ideomotor testing. There was no association between ideomotor, ideational or constructional apraxia and DAM. Six of the 10 DAM patients developed incontinence as part of their illness. Continence was regained in all of those patients who survived.

\section{Patients unable to walk on admission}

Of 16 patients ( $21 \%$ ) unable to walk independently on admission, only five were unable to walk even with assistance. Of these five, three were in the DAM group described in Table V. The other two patients were respectively diagnosed to have intractable vomiting and a history of falls with diuretic-related hyponatraemia. Of the 16 patients with loss of walking independence, four died $(25 \%)$ including two of the five who were immobile despite assistance on admission. The incidence of death during hospital stay was no greater in the $\mathbf{1 6}$ patients who were unable to walk on admission compared to the rest of the group.

Those who lacked independence in walking on admission had significantly higher disability as indicated by lower Barthel score (mean Barthel score of patients with loss of walking independence $=10.9$, s.d. 3.9 , compared to mean score of walking independent group $=18.0$, s.d. $2.7, P<$ 0.001 ).

A significantly greater proportion of the patients who lacked independent walking on admission had a low mental test score (less than $7 / 10)(P<0.01)$. By one week, eight of these $16(50 \%)$ patients had regained walking independence, including two of the five unable to walk even with assistance. Of the 70 patients discharged, only one patient failed to be independently mobile at discharge.

\section{Discussion}

In the acute medically ill elderly, acute deterioration in gait and balance is a feature recognized by many clinicians. However, the mechanism responsible for the acute changes in gait, posture and truncal movements is poorly understood. The group of elderly patients who are most susceptible to this marked change in functional ability has never been (as far as we are aware) identified before. Nor has there been a study attempting to assess the characteristics of gait and axial movement disturbances and relationships to eventual outcome in a cohort of elderly patients admitted with an acute medical illness.

Our observations suggest that, in the elderly, immobility with difficulty in postural adjustment unassociated with any deficit of upper limb coordination may accompany a primary acute nonneurological illness. The reversible nature of the disorder of gait and truncal movement as evidenced by independent walking in all but one survivors and high Barthel scores at discharge into the community, suggests that the expection for functional recovery is good.

The control group was not sex- or age-matched to the patient group. However, there was no association between the sex or age of the patient and independence in walking on admission, nor between those patients who scored above, or equal to or below five on axial movement scoring. A greater proportion of the patients in the DAM group, and those who lacked independent walking had a low mental test score. Although the mental test used does not discriminate between subjects with mental impairment due to a confusional state or dementia,${ }^{2}$ the admission score was significantly associated with that at discharge, suggesting that patients were maintaining their admission allocation. This study emphasizes that any assessment of locomotor and axial movement ability in patients with mental impairment must be certain that the presence of these signs is not a consequence of a coincidental medical illness.

By studying a control group of hospital visitors, we have been able to establish a range of functional ability and motor performance of the healthy elderly. For example, Horenstein suggested that normal elderly people should be able to stand on one leg for 10 seconds. This contrasts to our control 
population of elderly subjects, with only 16 of 90 subjects $(18 \%)$ able to do so.

We do not have a simple explanation for the reversible deficits described in our patient population. None of our patients underwent brain imaging nor were tests of vestibular function performed. Further studies using computed tomographic scanning and a neuro-otological assessment may help to define the nature of the disturbance causing the reversible deficits in mobility in our patients.

Transient adjustments in gait and axial movements may be an appropriate response in a patient who perceives an impairment of balance, in order to avoid further injury. Ring et al..$^{10}$ suggest that falls are more likely to occur in those subjects with a mild impairment of balance who have not developed protective strategies. However, there appears to be more than just the development of a cautious gait pattern to avoid falling in a few patients in our study. A small number are unable to sequence a

\section{References}

1. Sudarsky, L. \& Ronthal, M. Gait disorders among elderly patients. A study of 50 patients. Arch Neurol 1983, 40: 740-743.

2. Hodkinson, H.M. Evaluation of a mental test score for assessment of mental impairment in the elderly. Age Ageing 1972 , i: $233-238$.

3. Collin, C., Wade, D.T., Davies, S. \& Horne, V. The Barthel ADL Index: a reliability study. Int Disabil Studies 1988, 10: 61-63.

4. Wade, D.T. \& Collin, C. The Barthel ADL Index: a standard measure of physical disability? Int Disabil Studies 1988, 10: 64-67.

5. Geschwind, N. \& Damasio, A.R. In: Vinken, P.J., Bruyn, G.W. \& Klawans, H.L. (eds) Handbook of Clinical Neurology, vol. 45. Elsevier Science, Amsterdam, 1985, pp. 423432.

6. Brown, J.W. In: Thomas, C.C. Aphasia, Apraxia and Agnosia. Clinical and Theoretical Aspects. Springfield, Illinois, 1972, Ch. 9. patients in our study. A small number are unable to sequence a series of limb movements in order, for example, to walk even a few steps with assistance, or turn over in bed. The cause of this is not clear.

The reversible nature of the truncal and gait disturbance is striking. We can only speculate as to the cause. The gait and truncal abnormalities have many similarities to those seen in "lower body parkinsonism' due to vascular disease, ${ }^{11,12}$ and to those seen in hydrocephalus, Parkinson's disease and frontal lobe disease. Patients who scored poorly on the mental test score at admission appear to be a more susceptible sub-group. Further investigation is warranted to define the aetiology.

\section{Acknowledgement}

We are grateful for the statistical help of Dr M. Jahanshahi.

7. Cox, J.G.C., Pearce, I., Steiger, M.J. \& Pearce, J.M.S. Disordered axial movements in Parkinson's disease - a true apraxia? In: Rose, F.C. (ed) Current Problems in Neurology, vol. 6. John Libbey, London, 1987, pp. 71-75.

8. Romberg, M.H. In: Sieveking, E.H. (ed.) A Manual of the Nervous Diseases of Man, vol II. Sydenham Society, Londons 1853, pp. 395-401.

9. Horenstein, S. Managing gait disorders. Geriatrics 1974, 29 86-94.

10. Ring, C., Nayak, U.S.L. \& Isaacs, B. Balance function in elderly people who have and who have not fallen. Arch Phys Med Rehabil 1988, 69: 261-264.

11. Thompson, P.D. \& Marsden, C.D. Gait disorder of subcortical arteriosclerotic encephalopathy: Binswanger's disease. Movement Disorders 1987, 2: 1-8.

12. Fitzgerald, P.M. \& Jankovic, J. Lower body parkinsonism: evidence for a vasular etiology. Movement Disorders 1989, 4: $249-260$. 